\title{
The potential role of herbal medicines in the treatment of chronic stable angina pectoris: a review of key herbs, and as illustration, exploration of the Chinese herbal medicine approach
}

This article was published in the following Dove Press journal:

Botanics: Targets and Therapy

28 December 2012

Number of times this article has been viewed

Kylie A O’Brien ${ }^{1,2}$

Luis Vitetta ${ }^{3}$

'Deakin University, Melbourne, Victoria, Australia; ${ }^{2}$ Monash Medical School, Prahran, Victoria, Australia; ${ }^{3}$ The University of Queensland, School of Medicine, Centre for Integrative Clinical and Molecular Medicine at the Princess Alexandra Hospital, Brisbane, Queensland, Australia
Correspondence: Kylie O'Brien Deakin University, 22I Burwood Highway, Burwood 3125,Victoria, Australia Tel +6I $352278 \mid 46$

Email kylie.obrien@deakin.edu.au

\begin{abstract}
Herbal medicines have been used for centuries within different cultures to treat cardiovascular disease, including stable angina pectoris. However, the use of herbs varies within traditions of natural medicine, and how they are understood to work in systems such as Chinese medicine, for example, is vastly different from the pharmaceutical model that seeks to reduce herbs to their active constituents. This review first discusses, individually, key herbs used within Western, Indian, and Chinese herbalism to treat stable angina pectoris and their main active constituents and pharmacological actions. The second part of the paper then specifically explores how angina is treated traditionally with Chinese herbal medicine, a unique approach to the understanding of health and illness underpinned by philosophies and theories that describe the physiological functioning and pathological changes in the body in terms very different from those of biomedicine. A foundational account of the guiding theories of Chinese medicine is followed by a description of the cardiovascular system and the etiology and pathogenesis of angina from the Chinese medical perspective. This forms the basis for understanding the rationale for construction of Chinese herbal medicinal formulae for treating angina pectoris. The scientific evidence of the efficacy of some Chinese herbal formulae is discussed.
\end{abstract}

Keywords: herbs, herbal medicine, Chinese herbal medicine, angina pectoris, cardiovascular

\section{Introduction}

Phytomedicine can play a significant role in the treatment of cardiovascular disease (CVD), including treating mild forms of heart failure and coronary insufficiency, preventing and treating atherosclerosis and its sequelae, and treating symptoms of chronic venous insufficiency. ${ }^{1}$ Various herbs used to treat angina pectoris are found within the Western herbalism and Eastern herbal medicine traditions. However, the use of herbs varies within traditions of natural medicine, and how they are understood to work in systems such as Chinese medicine is vastly different from the pharmaceutical model that seeks to reduce herbs to their active constituents. Chinese medicine could be described as an energetic model of the mind-body, and it is underpinned by philosophies and theories that describe the physiological functioning and pathological changes in the body in terms very different from those of biomedicine. It is important to understand that there are paradigmatic differences between traditional medical systems and biomedicine that rest on different metaphysics or notions of what constitutes reality. 
Forms of herbalism such as Chinese herbal medicine (CHM) have developed on the basis of unique models of how the mind-body works.

In ancient Chinese medicine, what is recognized today as angina pectoris was grouped with a variety of other medical conditions under the broad clinical descriptor of "xiong bi" (chest obstruction), which has several underlying subcategories called syndromes. ${ }^{2}$ Herbal medicinal formulae are formulated for specific syndromes, composed of several herbs primarily chosen and judiciously combined according to Chinese medicine theory. In modern practice, Chinese herbs may also be chosen according to their (Western) pharmacological actions, but the way in which herbs are combined is essentially driven by traditional theory. The synergy between the herbs in medicinal formulae is likely to be the reason behind the efficacy of CHM.

In the first section of this review, key botanicals used to treat stable angina pectoris will be discussed individually, including their main active constituents and pharmacological actions. Some of these are used within Western herbalism, while others have traditionally been used in Chinese medicine and traditional Indian medicine (Ayurveda). The second part of the review then specifically explores how angina is treated traditionally with CHM. A foundation of the guiding theories of Chinese medicine leads into an exploration of how Chinese herbs are understood to work traditionally. A description of the cardiovascular system and the etiology and pathogenesis of angina from the Chinese medical perspective is given, providing foundational knowledge from which to understand the rationale for the making of Chinese herbal formulae to treat angina pectoris. The review concludes with a discussion of evidence from clinical trials relating to the efficacy of particular Chinese herbal medicinal formulae.

\section{Key herbs used in the treatment of stable angina pectoris}

Many botanicals within various herbal medicine traditions have been used to treat cardiovascular disease. These include hawthorn leaf and flower extract for heart failure and coronary insufficiency; garlic for atherosclerosis; Ginkgo biloba extracts for peripheral arterial occlusive disease; and horse chestnut extract for chronic venous insufficiency. Herbs such as Salviae miltiorrhizae Radix ("dan shen" in Chinese); Notoginseng Radix ("san qi"); Chuanxiong Rhizoma ("chuan xiong"); and Crataegi fructus ("shan zha") are common Chinese herbs used in the treatment of a wide range of cardiovascular conditions, including angina pectoris, though, in traditional use, they tend to be combined with other herbs. Although the role of some of these herbs may not be directly related to ameliorating the symptoms of angina pectoris, they might be beneficial secondary treatments for cardiovascular disease.

\section{Hawthorn}

Crataegus, commonly called hawthorn, is a large genus of shrubs and trees native to Europe, North America, and Asia that encompasses many species, including $C$. oxyacantha (in the West), C. monogyna (common hawthorn), C. laevigata, and $C$. pinnatifida (the Chinese herb shan zha). ${ }^{3}$ The leaves, flowers, and berries of hawthorn contain various bioflavonoids thought to be responsible for its actions on the heart. The main cardioprotective activity of $C$. oxyacantha is attributed in particular to its oligomeric proanthocyanidin content. ${ }^{4}$ The main active constituents include quercetin, quercetrin, triterpeeb saponins, vitamin $\mathrm{C}$, and cardioactive amines. ${ }^{4}$ The traditional herbal medicine use of the hawthorn berry has included angina pectoris, myocardial weakness, hypertension, tachycardia, arteriosclerosis, and Buerger's disease. $^{1}$

Several studies demonstrate the cardiotonic effects of hawthorn extracts, which include increasing the force of myocardial contraction, enhancing coronary blood flow, improving oxygen utilization by cardiomyocytes, and prolonging the refractory period and action-potential duration in heart and papillary muscles. ${ }^{3,5,6}$ Research has found that hawthorn can increase coronary perfusion, has a mild hypotensive effect, and antagonizes atherogenesis with a negative chronotropic effect and a positive inotropic effect on the contraction amplitude of cardiac myocytes. ${ }^{7-9}$ Hawthorn has been found to reduce incidence of reperfusioninduced ventricular fibrillations, ${ }^{5,10}$ reduce mortality rate, ${ }^{5}$ and decrease the mean duration of arrhythmias in ischemiareperfusion animal studies. ${ }^{10}$ The area of myocardial infarction was significantly smaller in rats treated with hawthorn extract, compared with controls. Zapatero ${ }^{11}$ provides a summary of studies that have investigated the potential mechanisms by which hawthorn may exert its effect on the cardiovascular system.

Randomized controlled trials (RCTs) of the efficacy of hawthorn extract have demonstrated increased physical exercise capacity and decreased resting heart rate and mean diastolic blood pressure during exercise in patients with dyspnoea, ${ }^{12}$ and increased exercise capacity and cardiac performance in patients with cardiac failure. ${ }^{13-17}$ Valli and Giardina ${ }^{18}$ report several other studies of hawthorn that have demonstrated improvement in symptoms associated with 
heart failure (such as fatigue) and improvement in measures of cardiac function. Meta-analyses have concluded that hawthorn extract confers significant benefits as an adjunct treatment for chronic heart failure, benefiting symptom control (such as shortness of breath and fatigue) and physiologic outcomes (maximal workload, exercise tolerance, cardiac oxygen consumption). ${ }^{19}$ Concomitant use of hawthorn with digitalis has been shown to significantly enhance the activity of the latter. ${ }^{20}$ An observational cohort study found that the efficacy of a homeopathic preparation of Cranolin (based on extracts of Crataegus and Spigelia anthelmia) in patients with mild heart failure was not inferior to commonly used ACE inhibitors or diuretics for 13 out of 15 variables chosen to assess efficacy. ${ }^{21}$

Recently, it was reported in an RCT that a hawthorn extract dose (1000 mg, $1500 \mathrm{mg}$, or $2000 \mathrm{mg}$ ) did not mediate dilation of brachial artery flow. ${ }^{22}$ The study suggested that if the berry extract had a blood pressure-lowering effect, it was likely to be mediated via a nitric oxide-independent mechanism. ${ }^{22}$ A study investigating the efficacy of a Camphor-Crataegus berry extract combination in treatment of orthostatic hypotension reported a hemodynamic stabilizing effect on arterial pressure in orthostasis. ${ }^{23}$ However, results need to be viewed with some caution, because the placebo treatment was 20 drops of a $60 \%$ alcohol solution alcohol is known to exacerbate orthostatic hypotension. ${ }^{24}$

In vitro experiments demonstrate an effect of hawthorn on ion channels in cardiac and smooth muscle cells, ${ }^{25}$ and as pointed out by Zapatero, ${ }^{11}$ this may be the mechanism by which hawthorn is able to decrease ventricular arrhythmias, slow heart rate, and decrease diastolic blood pressure. Hawthorn also appears to have antioxidant properties, ${ }^{26,27}$ and it can inhibit the formation of thromboxane A2 in vitro. ${ }^{28}$

\section{Garlic (Allium sativum)}

Garlic is a traditional herb believed to have originated in Central Asia and which has been extensively studied in cardiovascular disease, with demonstrated effects on atherogenesis, lipid metabolism, vascular resistance, fibrinolysis, and platelet aggregation. ${ }^{29-32}$ Approximately $65 \%$ of garlic is water. ${ }^{32}$ Its content is high in saponins, potassium, phosphorus, sulfur, zinc, and phenolic; moderate in selenium, Vitamin A, and Vitamin C; and lower in calcium, magnesium, sodium, iron, and B-complex vitamins. ${ }^{32}$ There is strong evidence from in vitro studies that garlic can reduce parameters associated with CVD; however, clinical trial evidence has been variable. ${ }^{32}$ Several clinical studies have indicated garlic can inhibit platelet aggregation in healthy participants and those with CVD; however, the evidence has been more variable in demonstrating an effect of lowering serum cholesterol, blood pressure, and oxidative stress. ${ }^{32}$ Some studies were conducted in China on the effect of garlicin in patients with unstable angina; ${ }^{33-35}$ however, limited information is available in English (abstracts only). An early study that compared patients with unstable angina randomized to receive intravenous garlicin $(60 \mathrm{mg} /$ day $)$ or nitroglycerin ( $5 \mathrm{mg} /$ day) for 10 days did not demonstrate a difference in the effective rate between the two groups ${ }^{33}$ (the subtherapeutic dosage of nitroglycerin used should be noted). In a later comparative study, ${ }^{35} 34$ patients received garlicin intravenously (60 mg/day), and 21 control patients received nitroglycerin (the dosage was not reported in the abstract, the only primary source in English for this study) over 10 days. The results demonstrated that the total effective rates of symptomatic and electrocardiographic improvement after garlicin treatment were $82 \%$ and $62 \%$, respectively, though the abstract of this Chinese journal article does not state the effective rate for nitroglycerin nor how the total effective rate was defined, limiting its usefulness. In addition, the study reported that plasma endothelin and blood sugar levels were markedly lowered in the cases with diagnosed hyperglycemia. ${ }^{35}$ The lack of detail available in English and the propensity for Chinese studies in herbal medicine to use endpoints not commonly used in Western countries, such as total effective rates (which are often defined in relation to percentage of patients achieving particular, defined endpoints, for example, in relation to symptoms or ECG results), limits the usefulness of such studies. Further studies are clearly required before a conclusion can be reached with respect to the usefulness of garlic in treatment of stable or unstable angina.

\section{G. biloba}

The seeds (nuts) and leaves of the herb G. biloba have a long history of use in Chinese medicine. ${ }^{36,37}$ In Chinese medicine, the seeds have been used over a longer time period than the leaves for the treatment of pulmonary disorders, including cough and asthma, alcohol abuse, and bladder inflammation. The leaves are used to treat heart and lung disorders, as well as skin infections. ${ }^{37}$ Standardized extracts of the leaves are used widely in Europe as a phytomedicine and in the United States as a dietary supplement. ${ }^{36,37}$ Primary active constituents in the leaves of G. biloba are flavonoid glycosides and ginkgolides (types of diterpines which are potent inhibitors of platelet-activating factor). ${ }^{36}$ The standardized extracts EGb 761 (24\% flavonoid glycosides, 6\% terpene lactones, 
and less than 5 ppm ginkgolic acid) and LI1370, approved by the German Commission E, have been widely used in Europe for the treatment of peripheral vascular disease. Other clinical applications have included treatments for coronary heart disease, cardiac angina, cerebral infarction, Alzheimer's disease, age-related dementias, and diabetic nephropathy. ${ }^{36,38}$ $G$. biloba leaf extract has several pharmacological effects, including antioxidant and cardioprotective effects. Mahadevan and Park report that the cardioprotective effects of the leaf extracts are via antioxidant activity, antiplatelet activity and increased blood flow via release of nitric oxide and prostaglandins ${ }^{37} \mathrm{~A}$ recent robust clinical study has reported, though, that G. biloba EGb 761 did not decrease CVD events or reduce mortality from CVD. ${ }^{39}$

\section{Arjuna (Terminalia arjuna)}

For centuries, the stem bark of T. arjuna (Roxb.) has been used by the ayurvedic traditional physicians in India for the treatment of various cardiovascular diseases. ${ }^{40,41}$ The bark of the species T. chebula Retz is also used in traditional Chinese medicine ("he zi pi" in Chinese).

Research in animal models has demonstrated T. arjuna to be positively inotropic, to have a vasodilatory effect on coronary arteries, and to have antioxidant and lipidlowering effects. ${ }^{41}$ Active constituents isolated from the stem bark include triterpinoids, flavonoids, and minerals. ${ }^{41}$ In a double-blind crossover study, $T$. arjuna extract was orally administered at a dose of $500 \mathrm{mg}$ 8-hourly, versus placebo for 2 weeks, to 18 healthy, normotensive, nondiabetic, normolipidemic, chronic cigarette smokers with no family history of CVD. ${ }^{42}$ T. arjuna was shown to reverse smoking-related endothelial dysfunction as measured by both endothelium-dependent and endothelium-independent, flow-mediated dilation of the brachial artery. There was no smoking interruption during the intervention, in order to remove any beneficial effect from smoking cessation that could confound the study results. An additional open-label study ${ }^{43}$ demonstrated a significant reduction in the frequency of angina episodes in those participants with stable angina pectoris, though study participants with unstable angina showed no significant improvement.

In an open-label study investigating the efficacy of Hartone, a preparation containing T. arjuna, 10 subjects with stable angina pectoris were given two capsules of Hartone twice daily for 6 weeks, then one capsule twice daily for the next 6 weeks. The efficacy of the herbal extract was assessed by reductions in the number of anginal episodes and improvement in a stress test. ${ }^{44}$ The results were compared with 10 patients with stable angina pectoris taking isosorbide mononitrate (ISMN) at an dose of $20 \mathrm{mg}$ administered twice daily. The study found that Hartone provided relief of symptoms in $80 \%$ of patients, compared with $70 \%$ taking ISMN, and that the number of angina attacks decreased from $79 /$ week to $24 /$ week in the Hartone group, compared with a reduction of 26/week to 7/week in the ISMN group. Both groups demonstrated improvements in several stress-test parameters, though there was no statistically significant difference between groups. Safety data from the study indicated that Hartone was better tolerated than ISMN and there was no evidence of renal or hepatic impairment.

A more recent RCT inducted 58 male subjects with chronic stable angina (New York Heart Association [NYHA] class II-III) with evidence of provocable ischemia on a treadmill exercise test. Participants received T. arjuna at an administered dose of $500 \mathrm{mg}$ 8-hourly or ISMN at a dose of $40 \mathrm{mg} /$ daily or a matching placebo for 1 week. ${ }^{45}$ Treatments were separated by a wash-out period of at least 3 days. The study showed that $T$. arjuna bark extract led to statistically significant improvement in clinical and treadmill exercise parameters $(P<0.005$ for four parameters $)$ and was associated with a statistically significant decrease in frequency of angina and need for isosorbide dinitrate $(P<0.005)$, in comparison to placebo. Similar improvements in clinical and treadmill exercise parameters were found for the ISMN therapy group, in comparison to placebo (as were found for T. arjuna). There was no significant difference between the T. arjuna and ISMN groups on these clinical and exercise parameters. The herbal extract was found to be well tolerated.

\section{Pushkarmoola (Inula racemosa)}

The root powder of I. racemosa, an herb used in ayurvedic medicine, is used to treat angina and dyspnea. ${ }^{6}$ Animal studies suggest a cardioprotective effect from this herb. ${ }^{6}$ Research in frogs isolated four major constituents, A, B, C, and D, with constituent $\mathrm{D}$ found to produce a negative ionotropic and negative chronotropic effect on frog heart. Constituent D was found to block the action of adrenaline and act as an agonist for propranolol. ${ }^{46}$ Research on Wistar rats treated with $I$. racemosa indicated improvements in antioxidant status, hemodynamic function, and left-ventricular function, in comparison with rats treated with placebo; this was thought likely mediated via suppression of oxidative stress. ${ }^{47}$

In a preliminary clinical study conducted almost 30 years ago, the anti-anginal effect of I. racemosa in comparison with nitroglycerin was investigated in nine patients with ischemic heart disease. Pretreatment with I. racemosa 
3 g administered orally 90 minutes prior to testing was associated with less marked post-exercise ST depression. ${ }^{48}$ It is impossible to confirm if the nitroglycerin was administered sublingually or orally, but if the latter, this could invalidate the results. Furthermore, in a small open-label clinical study of 60 subjects diagnosed with angina pectoris, I. racemosa $(\mathrm{n}=30)$ and Saussurea lappa $(\mathrm{n}=30)$ were each administered orally as $500 \mathrm{mg}$ capsules three times daily over 90 days. $^{49}$ Within-groups analysis indicated a statistically significant reduction in frequency of angina episodes at one month and three months for both I. racemosa and S. lappa, with the mean number of angina episodes reverting to pretrial levels one month after cessation of the herbal therapy for each of the herbs. ${ }^{49}$ Two standard species of the herb Inula Radix, Inula racemosa Hook $f$ ("zong zhuang tu mu xiang" in Chinese), and Inula helenium L. ("tu mu xiang") are used in Chinese medicine to treat sensations of fullness and distension in the chest and to alleviate pain. ${ }^{50}$

\section{Turmeric/curcumin}

Turmeric (Curcuma longa) is a rhizomatous herbaceous perennial plant of the ginger family. Curcumin is an extract of turmeric $(C$. longa/C. domestica) and temulawak (C. xanthorrhizae) ${ }^{38}$ and is responsible for the yellow color of turmeric. ${ }^{51}$ Active constituents of turmeric include the curcuminoids demethoxycurcumin (curcumin II), bisdemethoxycurcumin (curcumin III), and cyclocurcumin. ${ }^{51}$ In general, little is known about the effect of curcumin on CVD; however, there is increasing evidence that it has a cardioprotective role against many CVDs. Wongcharoen and Phrommintikul report curcumin has antioxidant effects that attenuate adriamycin-induced cardiotoxicity, may prevent cardiovascular complications associated with diabetes, and has antithrombotic, antiproliferative, antiinflammatory, and lipid lowering effects. ${ }^{51}$ They also report that animal model research has demonstrated that curcumin's ability to inhibit p300-HAT can ameloriate the development of cardiac hypertrophy and heart failure. Further, curcumin has been shown to have an antiproliferative effect, and its antiinflammatory effects may prevent atrial arrhythmias. ${ }^{51}$

An RCT conducted to evaluate the effects of curcumin on total cholesterol, LDL cholesterol, HDL cholesterol, and triglycerides in acute coronary syndrome patients demonstrated its efficacy. ${ }^{52}$ The study concluded that the administration of low-dose curcumin $(15 \mathrm{mg} /$ day, three times daily) was associated with a trend toward reduced total cholesterol and LDL cholesterol levels in acute coronary syndrome patients. Accordingly, turmeric may show efficacy as a secondary herb for angina pectoris. However, in general, more research is needed to establish its effect clinically on CVD.

\section{Dan shen (Salvia miltiorrhizae Radix)}

A key herb in the Chinese materia medica for the treatment of cardiovascular conditions is "dan shen" (the dried root of S. miltiorrhizae Bunge). Dan shen and its major known chemical constituents, tanshinone (I, IIA, and IIB) and salvianolic acids (A, B), have been shown to have anticoagulant, vasodilatory, antiinflammatory, free-radical scavenging, and mitochondrial-protective actions, and can increase blood flow. ${ }^{53}$ Dan shen is used to treat a variety of cardiovascular and vascular conditions, including angina, ischemic heart disease, heart failure, hypercholesterolemia, hypertension, cerebral atherosclerosis, diffuse intravascular clotting, thrombophlebitis, and thromboangiitis obliterans, though its use in CHM extends beyond the cardiovascular system. ${ }^{7,18,50,53-56}$

Dan shen has been shown to have cardioprotective and cardiotonic effects. ${ }^{57,58}$ The herb is able to enhance the recovery of contractile force upon reoxygenation after myocardial ischemia. ${ }^{57}$ In animal studies of myocardial ischemia, dan shen extract has been found to reduce ischemic symptoms and improve the pathological electrocardiogram (ECG) pattern. ${ }^{7}$ An herbal formula, compound Salviae miltiorrhiz injection, (containing S. Miltiorrhizae Radix, Lignum Dalbergiae odoriferae, and electrolytes), was found to markedly inhibit increases in the myocardial phosphatidylinositol-4,5-bisphosphate and inositol-1,4,5trisphosphate that occur during myocardial ischemia in rats, which led the authors to postulate that inhibition of the phosphoinositide message system may be a means by which the herbal medicine exerted a cardioprotective effect in myocardial ischemia. ${ }^{59}$

Salvianolic acid A and salvianolic acid B (also called lithospermic acid A and B) have been found to be protective against cerebral and myocardial ischemia. ${ }^{60}$ A negative inotropic effect and increased coronary flow rate were demonstrated in isolated rat hearts that were perfused with dan shen for 20 minutes, then made ischemic for 30 minutes, and then reperfused for 30 minutes, compared with untreated (control) hearts. ${ }^{61}$ Additionally, the recovery of left-ventricularly developed pressure in hearts treated with dan shen was significantly better, and contractions significantly less, than in untreated hearts during postischemic reperfusion. ${ }^{61}$ Other animal studies have indicated that active constituents can reduce the infarct size caused by 
myocardial ischemia. ${ }^{62,63}$ Salvianolic acid B was found to reduce myocardial damage in rabbit ischemia-reperfusion models, ${ }^{62}$ and tanshinone was found able to reduce the risk of myocardial tissue necrosis. ${ }^{7}$ Dan shen's ability to upregulate expression of vascular endothelial growth factors in human fibroblasts, generating new vascular endothelial cells, suggests dan shen's potential role in restoring myocardial function following myocardial ischemia. ${ }^{64,65}$

Dan shen dilates coronary arteries at all concentrations, but its actions on other blood vessels are variable, ${ }^{57}$ as are its effects on blood pressure: it causes general vasodilation and lowers blood pressure at low doses, and vasoconstriction in noncoronary arteries at higher doses. ${ }^{66,67}$ In rat experiments, salvianolic acid B has been found to lower blood pressure in a dose-dependent manner and to relax the mesenteric arterial bed in a concentration-dependent manner. ${ }^{68}$ Other studies have also demonstrated salvianolic acid B's ability to lower blood-pressure. ${ }^{53,60}$ Salvianolic acid B has been shown to inhibit angiotensin-converting enzyme ${ }^{69}$ and to increase nitric oxide production by endothelial cells. ${ }^{53}$ In vitro, salvianolic acid $B$ has been found to cause endothelium-dependent vasodilation in aortic strips. ${ }^{70}$

Salvianolic acids and rosmarinic acid (another active constituent) have been found to inhibit thrombosis, thromboxane B2 formation, and platelet aggregration. ${ }^{60,71,72}$ Dan shen can increase superoxide dismutase activity in platelets, thereby protecting against pulmonary embolism and inhibiting platelet aggregation. ${ }^{73}$ Dan shen and salvianolic acid $B$ have been shown to inhibit expression of circulating adhesion molecules, ${ }^{74}$ and along with salvianolic acid $\mathrm{A}$, have been shown to have antioxidant effects. ${ }^{63,75,76}$ The cardioprotective action of salvianolic acids has been found to be due not only to their reactive oxygen species scavenging abilities, but also to their ability to reduce leukocyteendothelial adherence and inhibit inflammation and metalloproteinase expression of smooth muscle cells, as well as indirectly to regulate immune function. ${ }^{77} \mathrm{~A}$ cardioprotective mechanism of salvianolic acids has been found to involve competitive binding of salvianolic acids to target proteins, interrupting protein-protein interactions. ${ }^{77}$ Dan shen's effect on blood lipids, reducing cholesterol and triglyceride levels, decreasing endothelial damage, and inhibiting lipid peroxidation, has also been demonstrated. .,63,78 $^{2}$

An RCT of coronary heart disease patients treated with tanshinone IIA indicated that the treatment group had improved cardiac symptoms and ECGs, though there were changes neither in what was termed blood viscosity, nor in fibrinogen or cardiac contractility, nor in compliance or inotropy. ${ }^{79}$ Adams et $\mathrm{al}^{53}$ report that in a 1979 Chinese study that was not randomized, blinded, or controlled, 108 patients with angina pectoris were treated with tanshinone IIA injected intravenously and their symptoms and ECG changes monitored. It was reported that in patients who responded within four treatments or less, the treatment had an overall effective rate of $86 \%$. The effects were similar to those from a compound dan shen formula. They also found that 10 out of 12 patients with acute myocardial infarction improved with tanshinone IIA treatment. ${ }^{80}$ The methodological problems associated with this study limit its usefulness, however.

\section{San qi (Notoginseng Radix)}

The Chinese herb "san qi" (Notoginseng Radix) is the root of notoginseng or pseudoginseng. In the Chinese materia medica, san qi is categorized as an herb that invigorates the blood (see Angina pectoris in Chinese medicine: etiology and pathogenesis section). ${ }^{50}$ Major constituents include saponines (ginsenosides), notoginsenosides, various organic acids (including acetic acid), dencichine, amino acids, and sugars. ${ }^{50}$

San qi has traditionally been used for its hemostatic effect on internal and external bleeding, and for its reduction of swelling and alleviation of pain from traumatic injuries (eg, sprains, fractures) ${ }^{50,54}$ It is also used to treat angina and coronary heart disease, and can relieve myocardial ischemia, lower cholesterol, help control bleeding, reduce myocardial oxygen consumption, and increase coronary blood flow. ${ }^{81,82}$

Animal studies have demonstrated that san qi can dilate coronary arteries. ${ }^{57}$ However, the effect of san qi on blood pressure is uncertain: it may cause vasoconstriction or vasodilation, depending on the blood vessel and the concentration. ${ }^{57}$ The saponins in san qi have been found to lower blood lipids, ${ }^{82}$ and in vitro research suggests that saponins may inhibit atherosclerosis by inhibiting the proliferation of aortic smooth muscle cells. ${ }^{83}$ The total saponins of san qi have also been found to have an antiinflammatory effect in rat acute inflammatory models. ${ }^{84}$

Trilinolein, another compound in san qi, has demonstrated concentration-dependent antioxidant effects. ${ }^{85}$ Beneficial effects of trilinolein include reducing thrombogenicity and arrhythmias and increasing erythrocyte deformability. ${ }^{85}$ Rat studies have demonstrated that trilinolein suppresses cardiac arrhythmias during oxygen-deprivation ischemia and reperfusion and has a protective effect on the myocardium. ${ }^{85}$

Research also suggests that san qi may be useful for retarding the progress of diabetic nephropathy and for 
improving microcirculation. ${ }^{86}$ Patients with early diabetic nephropathy treated with either Ticlopidine or san qi were found to have significantly reduced thromboxane $\mathrm{B} 2$ and lower $\mathrm{T} / \mathrm{K}$ ratios, along with significantly reduced urinary albumin, beta-2 microglobulin, and blood alpha microglobulin. ${ }^{86}$

\section{Chuanxiong Rhizoma}

Another herb from the category of herbs that invigorate blood in the Chinese pharmacopeia, "chuan xiong" is widely prescribed for treatment of cardiovascular disease in China. ${ }^{87,88}$ Chuanxiong Rhizoma is the dried root of Ligusticum chuanxiong Hort. There are another two Ligusticum species, L. sinensis Oliv. and L. jeholense Nakai et Kitag, listed in the Chinese pharmacopeia. ${ }^{89}$ The main active constituents of L. chuanxiong Hort include phalides (senkyunolide, Z-ligustilide, neocnidilide, 3-butylphthalide, and ligustilide dimers) and terpenes. ${ }^{88,90}$ L. chuanxiong Hort (Umbelliferae) is often prescribed in conjunction with nitric oxide donors for treating angina and other coronary heart diseases. ${ }^{91}$ The effect of chuan xiong on blood circulation may be due to active components senkyunolide $\mathrm{A}$ and ligustiliden. ${ }^{88}$

Research using isolated rat aortas has demonstrated a relaxation effect of extract of $L$. chuanxiong Hort on vascular rings, and a significant inhibitory effect against contractions induced by phenylephrine; the potentiation of relaxation is postulated to be related to nitric oxide. ${ }^{92}$ The vasorelaxation effects of two of its main active constituents, ligustilide and senkyunolide A, were investigated in isolated rat aortas, with both having similar relaxation potencies against contractions induced by a range of contractile agents, including phenylephrine and $\mathrm{KCl}^{87}$ The vasorelaxation effects were not affected by endothelium removal. ${ }^{87} \mathrm{~A}$ later study that investigated the interaction between the major constituent butylidenephthalide and nitric oxide donor sodium nitroprusside in isolated rat aortas found a synergistic interaction between the two, producing relaxation. The mechanism was related to enhancement of the sodium nitroprusside's effectiveness in producing relaxation under tone induced mainly by $\mathrm{Ca}^{2+}$ sensitization. ${ }^{91}$ Tetramethylpyrazine, another active constituent has been found to increase coronary blood flow and decrease myocardial contractile force in isolated guinea pig hearts and in mice. ${ }^{89}$

Tumor necrosis factor (TNF)-alpha may contribute to myocardial damage during ischemia reperfusion. Another study in isolated rat hearts concluded that the cardioprotective effect of ligustrazine is associated with a reduction in TNF-alpha content through inhibition of free radical production. ${ }^{93}$ Z-ligustilide and senkyunolide $\mathrm{A}$, two major active constituents of $L$. chuanxiong, were found to have inhibitory actions on TNF-alpha production and TNFalpha bioactivity in human monocytic cell lines, leading researchers to postulate that they might be the mechanism by which $L$. chuanxiong exerts its beneficial effects in the treatment of cardiovascular disease and other inflammatory disorders. ${ }^{94}$ Essential oil of chuan xiong has antioxidant and antipyretic effects, and Z-Ligustilide has demonstrated neuroprotective effects on brain ischemia in mice. ${ }^{88}$

Xiao and $\mathrm{Liu}^{89}$ reported a controlled and self-crossover clinical trial by $\mathrm{Hu}$ et al that was conducted in China in 218 patients with angina pectoris who were treated with Rhizoma Ligustici for 3 weeks. Results indicated a total effective rate of $90 \%$ in the treated group, ${ }^{89}$ though how this effective rate was defined is not explained. As mentioned previously, the use of terms such as "total effective rate" are common in Chinese clinical studies, but not in studies from Western countries.

\section{CHM and the treatment of angina pectoris}

In the previous section, individual herbs from Western, Indian, and Chinese herbalism that are used or show promise in the treatment of stable angina pectoris were discussed, with particular focus on research into the pharmacological actions of the herbs and some of their active constituents. This is, by nature, a reductionist approach to understanding herbs. In contrast, in traditional medical systems such as Chinese medicine, several herbs are typically combined in medicinal formulae to treat conditions such as angina and other cardiovascular conditions. It is likely to be the synergism between herbs or between active constituents that is responsible for their therapeutic actions. At a fundamental level; however, how CHM is practiced is underpinned by a different model or understanding of how the human body functions compared with the biomedical model.

Angina pectoris has been treated with CHM under the broad clinical descriptor "xiong bi" (chest obstruction), which covers several conditions affecting the cardiovascular, respiratory, musculoskeletal, and gastrointestinal systems that all have chest pain in common. ${ }^{2}$ In order to understand how CHM is used to treat angina, it is necessary to have some appreciation of the Chinese medicine system as a whole, including its key theories and how CHM in general is understood to work. This will be explored first, then consideration given to how the cardiovascular system and the etiology and pathogenesis of xiong bi and angina are understood in Chinese medicine. The rationale for combining 
herbs into medicinal formulae to treat angina pectoris emerges from this. The scientific evidence relating to clinical efficacy of various medicinal formulae will conclude this section.

\section{The Chinese medicine system}

Chinese medicine developed over two thousand years ago, based on metaphysics and a world view quite different from that underpinning biomedicine. Central to the understanding of how the universe existed and how the human functioned was the notion of "qi," a concept with many varying definitions. ${ }^{95}$ In simple terms, qi is the material basis for the human body and, simultaneously, an intangible motive life force or energy. ${ }^{96,97} \mathrm{Qi}$ is believed to circulate around the body in special pathways called meridians.

The philosophy of holism pervades many aspects of this medicine. The human is viewed within the context of her/his environment, interdependent and not separate. ${ }^{96}$ Environmental factors (such as wind, cold, and heat) can become pathogenic to humans. The holism between mind and body is evident in the classification of emotions as one of three important causes of disease in discussions on etiology. The body's organ systems are seen as functionally interdependent, and these relationships were described by particular theories (described later). Treatment, whether with acupuncture, CHM, diet therapy, or other modalities, is individualized, with several factors taken into account, including age, gender, individual constitution, and season of disease occurrence. ${ }^{96}$

A characteristic of Chinese medicine is the subcategorization of a disease (disease here being broad and inclusive of disorders, symptoms, and signs) into several (typically four to six) syndromes or patterns of disharmony, each of which is characterized by particular signs and symptoms indicative of the underlying etiology and pathogenesis of the condition at that point in time. ${ }^{98}$ Syndromes may change over time as a disease progresses, or as the patient begins to recover health. While one pattern may be predominant, it is not unusual to find combinations of syndromes. Treatment is focused on the underlying pattern of disharmony, or syndrome, of a disease. The herbs used to make up the medicinal formulae differ depending on the syndrome. ${ }^{98}$ This is a particular point of difference from biomedicine, which tends to prescribe pharmaceuticals on the basis of the disease. The process of differentiating syndromes involves analyzing clinical data collected during a Chinese medicine examination, according to several Chinese medicine theories, to arrive at a diagnosis of the disease and its underlying pattern of disharmony.
For example, a person with diabetes may be diagnosed with "Kidney and Stomach Yin deficiency," denoting that both the "Kidney yin" and "Stomach yin" are below par or deficient. Chinese herbs that specifically nourish the yin of the body, in particular those that target the kidney and stomach, will be included in the medicinal formula.

The causes of disease include environmental exogenous pathogenic factors (wind, cold, heat, summer heat, dampness, dryness) that, if they occur out of season or are particularly strong and the person is susceptible in relation to them, can become pathogenic to the body. ${ }^{96}$ Signs and symptoms in the body are described by way of analogy to these environmental factors. For example, signs or symptoms occurring rapidly and changing location in the body indicate the presence of wind; ${ }^{96}$ where there is sticky discharge from the body (eg, nasal discharge) or edema, this would indicate dampness. There are also internal causes of these pathogenic factors, generated by dysfunction of the internal organs. In addition, emotional factors are recognized as causes of disease. ${ }^{96}$ Each organ system in the body has a particular related emotion, and if these are prolonged or particularly strong or sudden, they can cause imbalance. Other etiological factors are noninternal and nonexternal factors that include poor diet, lack of exercise, overexercise, trauma, and pestilence, among others. ${ }^{96}$ Treatment is aimed at the root cause (Chinese "ben") of the disease, as well as the symptoms or manifestations (the "biao" or branches, to use the analogy of a tree), another point of departure from biomedicine.

\section{Key theories of Chinese medicine}

Chinese medicine has its own theories that are used to explain the workings of the mind-body, to characterize health and what happens in disease, as well as guide treatment. There are several key theories that underpin the practice of Chinese medicine. It is beyond the scope of this review to give a full explanation of these theories, and the reader is referred to other publications..$^{98,99}$ A brief explanation of some key theories is provided below.

\section{Yin and yang theory}

Yin and yang are opposite but complementary forces or states of a cycle. Yin and yang can transform into each other, but cannot exist alone. Believed to originate from peasants' observations of the changes in light and shadow on the face of a mountain as the sun traversed the sky, yang became synonymous with light, warmth, day, and activity; yin became associated with darkness, coldness, night, and rest. Yin and yang were applied in medicine to describe the 
body, its physiological functioning, and pathological processes in disease. ${ }^{100}$ For example, the upper half of the body is yang, the lower is yin; yin is the interior of the body in relation to the exterior yang. The organ systems (described later) were also envisaged to have a yin and/or yang aspect. Pathogenesis of disease incorporates yin and yang theory. For example, a deficiency of the yang leaves yin in relative excess, or vice versa, each with ensuing pathological consequences. ${ }^{96}$ An absolute excess of yang or yin in relation to the other can be a potential cause of illness. ${ }^{97}$ Health is conceptualized as a balance of yin and yang, and illness as a loss of balance. Acute, rapid-onset diseases were seen as yang, while chronic diseases with slow progression were considered yin.

Applied to diagnosis, diseases exhibiting signs and symptoms such as a red face, fever, and sore throat were seen as suggestive of heat and therefore classified as yang in character. Diseases characterized by a subjective feeling of cold, cold extremities, pale tongue, white tongue coating, and pale complexion would be classified as cold in nature and therefore yin.

\section{The Five Element (Five Phase) Theory}

The Five Element Theory describes the relationship between elements of nature (fire, earth, water, metal, and wood) and draws analogies between the characteristics of these elements and the main organ systems in the body, sense organs, seasons, colors, tastes, sounds, and emotions. This theory describes the interrelationship between the five elements and therefore between organ systems, and is used to guide diagnosis and explain physiological functioning and pathology in disease. ${ }^{96}$

\section{Zang Fu Theory}

Zang Fu Theory is the main theory by which to describe the organ systems of the body, which are better thought of as functional systems. There are five "solid" ("zang") organs (the Heart, Lung, Liver, Kidney, and Spleen) that are linked with "hollow" ("fu") organs via the meridian system. (Note the names of the zang fu organs are capitalized to denote the Chinese medical concept of these organs, as opposed to the biomedical definition, throughout this paper.) The functions of the zang and fu organs diverge and extend beyond the biomedical function conceptually (and in some cases, there is no correlation). For example, the Spleen/Stomach pair is responsible for the digestion of food, which is converted into nutrient qi that circulates via the meridians and blood to nourish the other organ systems and body parts. ${ }^{97}$ This qi also forms part of blood, as understood in Chinese medicine theory. Particular signs and symptoms are characteristic of pathology of the zang fu organs. The Kidney, for example, "rules" the lower back, and its related sense organ is the ear, which is responsible for water metabolism; lower back pain problems, urinary dysfunction, and poor hearing are typically ascribed to a Kidney deficiency of some kind (ie, of qi, yin, or yang). ${ }^{96}$ Zang Fu Theory describes the functions of the various organs both as single organs and in relation to each other, and provides the theoretical basis for treatment. For example, if Lung yin is found to be deficient, since Kidney yin is known to be the basis of the yin of the whole body, the Kidney yin may also be deficient. Thus, in treating with CHM, herbs will be chosen that nourish both Lung yin and Kidney yin.

\section{Meridian Theory}

Meridian Theory describes how qi circulates in the body in special pathways, termed "meridians" or "channels." There are 14 main meridians in total, including one named after each of the zang fu organs, each of which passes through the organ, its paired organ, and its related-sense organ; and two meridians running along the body's midline dorsally and ventrally. There are also branches of the meridians (termed "collaterals"). This system of energetic pathways connects all aspects of the body, forming an integrated whole. It is the basis for understanding how the pathology in one organ may affect that of another.

\section{The Eight Guiding Principles}

The Eight Guiding Principles ("ba gang") are given in a set of four pairs of principles that serve to broadly categorize Chinese medicine syndromes: yin/yang, cold/heat, interior/ exterior, and deficiency/excess. Yin and yang are overarching summary principles: cold, interior, and deficiency conditions are yin, and heat, exterior, and excess conditions are yang. ${ }^{97}$ The cold/heat pair describes the inherent nature of the condition with respect to temperature. The interior/exterior pair denotes the location of the pathology - either in the superficial/exterior layers of the body or the interior (the majority of diseases). The deficiency/excess pairing relates, respectively, to the state of the patient's antipathogenic qi (the patient's defensive mechanisms) and to the strength of the pathogen. ${ }^{96,101}$ In an "excess" syndrome, the pathogen is abundant or predominant, but the patient's antipathogenic qi is not compromised. ${ }^{101}$ In a deficiency pattern, the patient's antipathogenic qi is deficient or below par, perhaps due to overwork, prolonged stress, illness, or poor diet. ${ }^{96}$ 
Signs and symptoms of a deficiency of qi, for example, include a weak pulse, weak voice, shortness of breath, and lack of energy. ${ }^{2,96}$ Typically, deficiency-type illnesses are chronic and slow to progress, and treatment is aimed at rectifying the deficiency (whether it is in qi, yin, or yang) with herbs that tonify and nourish, while excess-type illnesses are acute, with a rapid onset, and treatment is aimed at eliminating the pathogenic factor.

\section{How CHM works}

While the history of Chinese medicine begins during the Eastern Han Dynasty (25-220 CE), it is recognized that the Chinese used medicinal substances for millennia prior to this. ${ }^{50}$ The earliest recorded materia medica, the Classic of the Materia Medica (Shen Nong Ben Cao Jing), written no earlier than the first century CE, contained 364 entries (botanical, mineral, and zoological), with descriptions of each herb in terms of its properties and therapeutic actions, and any toxicity noted. Several hundreds of years later, subsequent materia medicas began to link classical theory to empirical knowledge of herbs. ${ }^{50}$

The taste and temperature properties of herbs help determine their therapeutic function. The five tastes are spicy or acrid, bitter, sweet, sour, and salty. Bland is a sixth taste. For example, bitter herbs are said to drain and dry and are often used in conditions where there is dampness. Sweet herbs harmonize and moisten and are typically used to tonify the qi, blood, yin, or yang. ${ }^{50}$ The temperature characteristics (the four qi) are cold, cool, warm, and hot (though a fifth category, neutral, is also used). Herbs that are warm/hot are used to treat cold syndromes; herbs that are cool/cold are used to treat syndromes where there is heat. Chinese herbs are said to enter particular meridians. Since the meridians are connected with particular zang fu organs, this provides the mechanism by which particular herbs target particular organs and/or have a particular action. ${ }^{50}$

In a modern Chinese materia medica, herbs are categorized in terms of their primary therapeutic action, though most herbs have at least three actions. Examples of herb categories include herbs that clear heat and relieve toxicity (these herbs typically have antipyretic and antimicrobial actions and are cold in nature), herbs that regulate qi (used in conditions where the qi is considered stagnant), and herbs that regulate blood (often used to treat pain and gynecological disorders). ${ }^{50}$

In CHM, herbs are combined according to particular principles to increase the therapeutic action of a formula, minimize toxicity or side effects, and/or accommodate complex clinical presentations. ${ }^{50}$ Certain combinations of herbs are contraindicated, as they may give rise to toxic side effects. Hundreds of classical Chinese medicinal formulae have been developed over the past 2000 years. Practitioners may use or modify classic formulae, or may develop their own medicinal formulae from first principles, based on an understanding of the etiology and pathogenesis of the disease and the syndrome diagnosis. CHM formulae are typically individualized and are often altered at subsequent visits as the clinical picture changes.

While CHM is primarily based on Chinese medical theories, in modern practice, practitioners may also select herbs based on a specific pharmacological effect on the body. The chemical constituents of herbs are well-established and pharmacological properties of Chinese herbs well researched. However, modern pharmaceutics seeks to reduce single herbs to key active constituents, with the aim of developing a patentable drug, an approach that is inherently reductionist. The efficacy of CHM is believed to reside with the combination of herbs, judiciously chosen according to the disease, and the underlying pattern of disharmony. Each herb may have several active constituents that, in combination with active constituents of other herbs, may have a synergistic effect. This is likely to be the reason for the efficacy of CHMs. It is important that researchers are cognizant of the practice of CHM.

\section{How Chinese medicine understands the cardiovascular system}

To begin to grasp how angina pectoris is understood and treated in Chinese medicine, an appreciation of how the cardiovascular system is understood in Chinese medicine is necessary. The Heart, as the "commander of blood," is the major organ responsible for regulating blood circulation, with the Heart qi providing the motive force for blood circulation. However, other zang organs also play a role. ${ }^{99,100}$ The relationships between the Heart and other organs are described using various theories, including the zang fu Five Element Theory and meridian theory (a number of the meridians and their smaller branches pass through the Heart, including the Heart, Kidney, and Spleen meridians and the Small Intestine meridian, the Heart's paired fu organ). ${ }^{102}$ The Lung, as the commander of qi, is responsible for qi circulation, and qi is a component of blood; thus, there is a close relationship between the Heart and Lung. The Spleen/ Stomach (zang fu paired organs) converts food to nutrient qi, which ultimately forms part of blood. The Spleen, via its holding function, ensures that blood does not extravasate 
from blood vessels. Thus, if the Spleen qi is deficient, the Heart qi may also become deficient (since qi is created by the Spleen for the whole body). The Heart blood may also become deficient (since qi forms part of blood). The Liver plays a role in the cardiovascular system via one of its chief functions: that of flowing qi freely around the whole body. Its role is storing blood (regulating the blood volume). If Liver qi is stagnant, Heart qi may also stagnate. Qi stagnation can lead to blood stagnation, when blood circulation is retarded. The Kidney is involved via its role in fluid metabolism and in particular is due to the relationship between the Kidney and Heart, which keep each other in check. ${ }^{99}$

The Heart is said to be the king of the emotions, describing its central role in controlling emotions (though each of the five zang organs is associated with a particular emotion). A more radical departure from the biomedical understanding of its function is that the Heart is responsible for housing the "shen," which refers broadly to spirit, or more narrowly to mental consciousness or activities, including memory, perception, and thinking. ${ }^{100}$ Thus, mind and consciousness are ascribed to the Heart (rather than the brain). Consequently, in addition to various cardiovascular conditions, pathology involving the Heart may also manifest as insomnia, poor memory, and excessive dreaming. ${ }^{99}$

\section{Angina pectoris in Chinese medicine: etiology and pathogenesis}

Traditionally, xiong bi (also called chest bi [obstruction]) was used to cover any condition that manifested as pain, discomfort, fullness, or a feeling of oppression in the chest region. Xiong bi includes cardiovascular conditions such as angina pectoris and myocardial infarction, as well as other biomedical conditions affecting the lungs, musculoskeletal system, and gastrointestinal system. The basic common mechanism of chest pain, regardless of cause, is obstruction of the circulation of qi and blood. ${ }^{2}$ The underlying pathogenesis of chest pain may be one of deficiency or excess (see earlier discussion on the Eight Guiding Principles): deficient qi fails to promote blood circulation and deficient yin causes increased blood viscosity, while an excess pathogen blocks qi and blood circulation. In terms of the etiology of xiong bi, typically it is a mixture of both deficiency and excess, with the root cause being a deficiency in qi, yin, and/or yang deficiency; the manifestations are then excess (due to phlegm stagnation, cold stagnation, heat, qi stagnation, or blood stagnation). ${ }^{2}$ Thus, the idea of blockage is involved in xiong bi, conceptually understood in Chinese medicine as blockage of the Heart or Heart orifice. Xiong bi has several patterns of disharmony, each of which is characterized by particular signs and symptoms and each of which requires the formulation of different CHMs. The Chinese medicine practitioner must, through a careful case history and examination of the patient, ascertain the root cause and the underlying pattern(s) of disharmony before creating the CHM formula.

In modern Chinese medicine, there is an increasing trend to apply the process of differentiation of syndromes to specific, biomedically defined clinical conditions or diseases to identify their specific underlying patterns of disharmony, though appropriately designed empirical studies on which to base such assertions are often lacking. ${ }^{103}$ In general, the Chinese literature suggests that the main patterns associated with angina pectoris are qi and yin deficiency, blood stasis, qi stagnation, and phlegm stasis. ${ }^{104,105}$

Blood stasis describes the condition in which the local or systemic blood circulation is retarded, a concept developed in particular during the Qing Dynasty. ${ }^{96}$ The clinical manifestations of blood stasis are pain (typically in a fixed location and typically with a stabbing quality), dark and purple lips, dark complexion, dry scaly skin, irritability, purple tongue body, masses in the body (eg, tumors), ecchymosis, clots in menstrual blood, and telangiectasia. ${ }^{96}$ Blood stagnation may be caused in several ways. For example, if qi is deficient, it will not provide sufficient motive force for blood circulation. If qi is stagnant, blood flow will likewise be retarded. When there is blood stasis, qi is also stagnant, as qi provides the motive force for blood circulation, though the converse is not always true. When Chinese herbs are chosen to treat blood stagnation, the root cause of the stagnation must also be treated. If blood stasis is due to qi deficiency, herbs that tonify qi will be chosen, in addition to herbs that invigorate blood (promote blood circulation). Qi stagnation in the Heart meridian typically manifests as a distending type of pain and is often associated with emotional factors. ${ }^{96}$ Qi stagnation can affect blood circulation and combine with blood stasis and/or phlegm retention. ${ }^{96}$ Where there is qi stagnation, herbs that regulate qi are included in the medicinal formulae.

The CHM concept of phlegm ("tan" in Chinese) is perhaps more difficult to reconcile with biomedicine. In classic literature, phlegm was defined pathophysiologically as a process of condensation and congealing. ${ }^{106} \mathrm{Phlegm}$ is envisaged as thick and viscous, while phlegm-fluid retention ("tan yin") is a thin, clear, light fluid in a fixed location. ${ }^{107}$ In modern Chinese medicine, a distinction is made between tangible phlegm that can be seen (eg, nasal discharge), and intangible phlegm, the presence of which is deduced 
from various signs and symptoms (such as dizziness and palpitations), though in all cases, it is due to some kind of disturbance to fluid metabolism as understood in Chinese medicine. ${ }^{107}$ The patient's presenting signs and symptoms are indicative of where the phlegm has stagnated or is blocking. For example, phlegm blocking the Heart may produce a suffocating sensation in the chest, such as during an angina or heart attack, plus palpitations. ${ }^{96}$

\section{CHM treatment of angina pectoris}

To treat angina, CHMs are created to address the underlying pattern(s) of disharmony diagnosed. Thus, in the case where qi and yin are deficient, herbs from the categories of herbs that tonify qi and herbs that tonify yin would be chosen from the Chinese materia medica. Where there is blood stagnation, herbs from the category of herbs that invigorate blood are chosen. Where qi is found to be stagnant, herbs from the category of herbs that regulate qi are chosen. If phlegm is present, then herbs are chosen that dry dampness (phlegm is understood to be a form of dampness) and resolve phlegm, with other herbs chosen to address the root cause of the phlegm in the first instance. Different herbal formulae may be chosen or constructed at different times depending on the patient's presentation. For example, in general, particular herbal formulae are chosen to treat the acute presentation of angina pectoris, aimed at treating the biao/branch/manifestation - an excess-type pattern of disharmony, while other formulae will be used after the acute presentation has passed that are focused on addressing the root cause (ben) which is a deficiency pattern (which may be deficiency of qi, yin, or yang). These formulae will contain quite different herbs.

There are many Chinese herbs used to treat angina pectoris. The rationale for using these herbs in angina treatment not only comes from within the theory of Chinese medicine but also from knowledge of their pharmacological actions, derived from research. For example, as reported by Zhuo and colleagues, ${ }^{108}$ several herbs relieve myocardial ischemia by dilating the coronary arteries and increasing coronary blood flow, such as chuan xiong (Ligustici Chuanxiong Rhizoma), dan shen (S. miltiorrhizae Radix), san qi (N. Radix), hong hua (Carthami Flos), ge gen (Puerariae Radix), and huang qi (Astragali seu hedysari Radix). ${ }^{81,108}$ Others reduce myocardial oxygen consumption, such as dang gui (Angelicae sinensis Radix) and san qi (Notoginseng Radix). Still others have antiplatelet and anticoagulant activity, acting on blood clotting factors, increasing level of cAMP in thrombocytes, and inhibiting the rheological state of blood to inhibit thrombi formation, such as dan shen (S. miltiorrhizae Radix).
Some herbs such as dan shen (S. miltiorrhizae Radix), san qi (Notoginseng Radix), and dang gui (Angelicae sinensis Radix) can lower blood lipids and thereby counter atheroma development. Other herbs are used to treat pain associated with angina, such as dang gui (Angelicae sinensis Radix), san qi (Notoginseng Radix), and huang qi (Astragali seu hedysari Radix). ${ }^{81,108}$ The majority of these herbs are categorized as herbs that invigorate blood in the Chinese materia medica. Four commonly used herbs are dan shen (S. miltiorrhizae Radix), shan zha (C. fructus), chuan xiong (Chuanxiong Rhizoma), and san qi (Notoginseng Radix), discussed in the first section of the review. Herbs from the category of aromatic substances that open the orifices, where sensory orifices include the Heart (remembering that the Heart is envisaged as the seat of consciousness), are often added to medicinal formulae and used in the treatment of angina pectoris. ${ }^{50}$ One such example is the herb shi chang pu (Acori tatarinowii Rhizoma), which can resolve phlegm and open the orifices, and is therefore useful where the Heart is said to be blocked, in the Chinese medical sense, by phlegm. ${ }^{50}$

An example of a key medicinal formula used to treat angina (and that may be modified by practitioners) is the formula named Modified Xue Fu Zhu Yu Tang. To illustrate the theoretical logic behind the construction of a medicinal formula, Table 1 sets out an explanation of the components of formulae ${ }^{109}$ and the rationale for their inclusion. This formula is focused on addressing an excess-type pattern of disharmony and would be more appropriate for treatment when angina attacks are occurring, that is, during the acute phase. Later, other medicinal formula focused on addressing the underlying root cause (ben), which is a deficiency pattern (of qi, yin, or yang).

\section{Clinical evidence of CHM's efficacy in treating angina}

In China, it is common belief that CHM is beneficial in the treatment of stable angina pectoris, in comparison with standard Western medicine. ${ }^{108}$ Numerous studies conducted in China have assessed the efficacy of CHMs in the treatment of angina pectoris, several with positive results. However, the methodological quality of many studies has been criticized. ${ }^{110}$ A 2010 Cochrane Review of traditional Chinese herbal products for stable angina identified 242 potentially relevant publications, which were reduced to 127 RCTs. ${ }^{108}$ Of these, only three finally met the inclusion criteria, but even these suffered from methodological flaws. For example, none reported methods to ensure compliance, one study was single-blinded (patients), only one reported withdrawals and 
Table I Herbal formula modified Xue Fu Zhu Yu decoction components ${ }^{109}$

\begin{tabular}{|c|c|}
\hline Herb & Rationale \\
\hline Tao ren (Semen Persicae, & Invigorates blood and removes \\
\hline peach kernel, Persica) & blood stasis \\
\hline $\begin{array}{l}\text { Hong hua (Flos Carthami tinctorii, } \\
\text { safflower flower, Carthamus) }\end{array}$ & $\begin{array}{l}\text { Invigorates blood and removes } \\
\text { blood stasis }\end{array}$ \\
\hline $\begin{array}{l}\text { Dang gui (Radix Angelicae Sinensis, } \\
\text { Chinese angelica root, tang-kuei) }\end{array}$ & $\begin{array}{l}\text { Invigorates blood and removes } \\
\text { blood stasis; tonifies blood and } \\
\text { moistens dryness }\end{array}$ \\
\hline $\begin{array}{l}\text { Chuan xiong (Radix Ligustici } \\
\text { Chuanxiong, Szechuan lovage root, } \\
\text { Enidium) }\end{array}$ & $\begin{array}{l}\text { Invigorates blood and removes } \\
\text { blood stasis }\end{array}$ \\
\hline $\begin{array}{l}\text { Chi shao (Radix Paeoniae Rubrae, } \\
\text { red peony root) }\end{array}$ & $\begin{array}{l}\text { Invigorates blood and removes } \\
\text { blood stasis; cools blood }\end{array}$ \\
\hline Niu xi (Radix Achyranthis & Improves circulation by \\
\hline Bidentatae, Achyranthes root) & $\begin{array}{l}\text { removing blood stasis and } \\
\text { inducing a downward movement } \\
\text { of blood }\end{array}$ \\
\hline Chai hu (Radix Bupleuri, & Regulates liver qi flow, raises \\
\hline Harea ear root, thorowax root) & $\begin{array}{l}\text { clear yang qi; by promoting } \\
\text { qi movement, blood circulation } \\
\text { is aided }\end{array}$ \\
\hline $\begin{array}{l}\text { Jie geng (Radix Platycodi Grandiflori, } \\
\text { balloon flower root, Platycodon) }\end{array}$ & $\begin{array}{l}\text { Regulates the flow of qi, } \\
\text { expands the chest, directs herbs } \\
\text { to upper jiao; by promoting } \\
\text { qi movement, blood circulation } \\
\text { is aided }\end{array}$ \\
\hline $\begin{array}{l}\text { Zhi ke (Fructus Citri Aurantii, bitter } \\
\text { orange ripened fruit) }\end{array}$ & $\begin{array}{l}\text { Regulates the flow of qi, } \\
\text { expands the chest; by } \\
\text { promoting qi movement, } \\
\text { blood circulation is aided }\end{array}$ \\
\hline $\begin{array}{l}\text { Sheng di huang (Radix Rehmanniae } \\
\text { Glutinosae, Chinese Foxglove root, } \\
\text { rehmannia) }\end{array}$ & Clears heat and cools blood \\
\hline $\begin{array}{l}\text { Gan cao (Radix Glycyrrhizae } \\
\text { Uralensis, licorice root) }\end{array}$ & $\begin{array}{l}\text { Regulates and harmonizes the } \\
\text { actions of the other herbs }\end{array}$ \\
\hline
\end{tabular}

the reasons for them, none described their exclusion criteria or allocation concealment, although randomization was reported, and none assessed quality of life. The conclusion drawn was that there was insufficient evidence to draw a conclusion about the usefulness of Chinese medicinal formulae for the treatment of stable angina pectoris, due to the small number of RCTs and small numbers of participants. They also concluded that there were insufficient data on safety. ${ }^{108}$

The Cochrane Review ${ }^{108}$ reported on several studies, as follows. In a single-blinded study by Fang et al, ${ }^{111}$ an herbal product, Yixinmai Granules, was compared with dan shen tablets. The Yixinmai Granules were more effective than the dan shen tablets (RR 2.71, 95\% CI 1.34-5.48) in terms of marked improvement in angina symptoms (defined as symptoms disappearing or being reduced by more than $80 \%$ ), though there was no significant difference between the medicines with respect to general improvement of angina symptoms (defined as severity, frequency, and duration of angina attack being reduced by $50 \%-80 \%$ ). In Wang et al's study, ${ }^{112}$ comparing the efficacy of a Bao Xin Bao patch with isosorbide dinitrite, the Bao Xin Bao patch was associated with a "marked improvement" in angina symptoms (RR 4.28, 95\% CI 1.60-11.39), but there was no difference between the two groups for "general improvement" of angina symptoms. In comparing the two medications using the criteria of "inefficacy" (defined as a reduction in the severity, frequency, and duration of angina attacks similar to before the intervention or reduced by less than $50 \%$ ), the results suggested that the Bao Xin Bao patch was inefficient. The number of patients with decreased use of nitroglycerin per week (by $50 \%$ or more) was significantly greater in the Baoxinbao patch group than in the isosorbide dinitrite group. The study found that the association of nitrates with general improvement in ECGs was of borderline statistical significance (RR 1.80, 95\% CI 1.02-3.17). In the third study by Wang et al, ${ }^{113}$ the Linaoxin Capsule was no more efficacious in terms of marked improvement or general improvement of angina symptoms than was the Xinnaokang Capsule, and there was no difference between the groups with respect to data on the inefficacy of interventions for improving angina symptoms. All studies reported ECG improvement but could not demonstrate a marked improvement. ${ }^{108}$

The difficulty in interpreting the results of the studies by Fang et $\mathrm{al}^{111}$ and Wang et $\mathrm{al}^{113}$ is that they used other herbal medicines as the control - dan shen preparations - rather than placebos, probably because the efficacy of dan shen in the treatment of ischemic heart disease is generally accepted. ${ }^{108}$ None of the studies used primary endpoints such as mortality figures or acute myocardial infarction. ${ }^{108}$

Another meta-analysis ${ }^{114}$ and a subsequent quality assessment was conducted comparing dan shen di wan (a Salvia compound pellet, CSP) with nitrates in the treatment of stable angina. Wang concluded that CSP significantly improved angina symptoms (RR 1.13, 95\% CI 1.07-1.20) and ECG results (RR 1.39, 95\% CI 1.28-1.50) and was associated with fewer adverse events, in comparison to nitrates. ${ }^{114}$ Unfortunately, the reliability of the studies was called into question because none of the studies had been reviewed by a human research ethics committee and none had obtained informed consent. ${ }^{108}$

The results of several RCTs suggest that some medicinal formulae may be efficacious in treating angina, ${ }^{108,115-120}$ though not all studies are methodologically rigorous and many compared the Chinese formula in question with 
another CHM. This is problematic if the efficacy of the comparator has not been established rigorously. In one double-blind, placebo-controlled RCT of a medicinal formula, Purified Coronary Heart II Tablets (containing five herbs, including dan shen), in 112 angina patients, improvements were recorded in terms of clinical symptoms and ECGs. The study reported that the Chinese herbal formula was $80 \%$ effective in comparison to placebo. ${ }^{119}$ In a study of poorer quality (noncontrolled, nonblinded, nonrandomized), 56 patients with angina or angina associated with previous myocardial infarction were intravenously treated with a dan shen formula for 14-30 days, in conjunction with standard pharmaceutical drugs. Patients were evaluated in terms of ECG changes and clinical symptoms. The study found an $89 \%$ effectiveness rate for improving angina paroxysms and a $67 \%$ effectiveness rate for improving heart conductance, as measured by ECG. ${ }^{120}$

In a single-blinded study over 6 months, 93 patients with stable angina were divided into three treatment groups: one taking Tie-Tao's Coronary Heart Disease Capsules, one group taking Compound Prescription Danshen Droplet Pills, and one taking isosorbide dinitrate (control). ${ }^{117}$ Patients recruited into the study had the Chinese medicine syndrome of qi deficiency, with blood and phlegm stasis. The groups showed comparable markedly effective rates in terms of reduced angina symptoms (chest pain gone or almost gone, 32\% vs $26 \%$ vs 39\%) and ECG changes (ECG normal or almost normal, $23 \%$ vs $19 \%$ vs $26 \%$ ) with no significant differences between groups, though the nitroglycerin usage per week was statistically significantly lower in the Coronary Heart Disease Capsules group compared to the other two groups at week $24(P<0.05){ }^{117}$

\section{Conclusion}

There are a range of herbs used within Western herbalism, Indian herbal medicine, and CHM to treat stable angina pectoris. This review has reported on several botanicals used in the treatment of stable angina pectoris. Varying degrees of scientific evidence exist and many appear to be very promising. Traditional use of herbs is a legitimate form of evidence, and medical systems such as Chinese medicine and ayurvedic medicines have a long history of traditional use of herbs. In relatively recent times, with the rise of the evidence-based medical approach, the emphasis with respect to evidence in medicine has shifted to scientific evidence, with RCTs and meta-analyses at the top of the hierarchy. When we examine evidence of the safety and efficacy of herbal medicines, the inherent limitations of RCT methodology need to be kept firmly in mind. When we examine herbs used within traditional medical systems, we also need to be very aware of the context in which they are used.

Chinese herbs are typically used as combinations of herbs, thoughtfully chosen according to an understanding of the etiology and pathogenesis occurring in the body at that point in time in that individual, within the theoretical framework of Chinese medicine. The potential synergistic effect of the herbs within a formula may be a key reason for its efficacy. The same logic applies to other traditions of herbal medicines, such as ayurveda. Therefore, to apply a Western reductionist approach, seeking only to understand herbs in terms of chemical constituents and pharmacological activities and isolating active constituents in the hope that one or two may prove to be "magic bullets," may be to miss the very reason for CHM's potential efficacy. In essence, forms of herbalism such as CHM and Indian herbal medicine seek to bring the human being back into balance and to address the root cause of illness, not simply target an offending pathogen or specific pathology (defined in biomedical terms). That is not to say that herbs are not used for their specific pharmacological effects in a Western sense, but in the case of Chinese medicine, for example, it is not the predominant method for constructing a medicinal formula - traditional theory guides this. The body has its own inherent defense mechanisms and healing abilities that traditional forms of medicine seek to tap into. Australian research indicates that the more common reason for use of medicinal herbs is health enhancement, though a high proportion of practitioners use herbs for a specific medical condition. ${ }^{121}$ This suggests, at least in that cohort, that people seek a state of health beyond just the absence of disease. A look into medical systems outside of Western medicine that are couched in terms of energetic models of the body provides a different perspective on what health is, what human beings are, and how they function.

\section{Disclosure}

The authors report no conflicts of interest in this work.

\section{References}

1. Fugh-Berman A. Herbs and dietary supplements in the prevention and treatment of cardiovascular disease. Prev Cardiol. 2000;3(1):24-32.

2. Maclean W, Lyttleton J. Clinical Handbook of Internal Medicine Volume 1: Lung, Kidney, Liver, Heart. Sydney, Australia: University of Western Sydney; 2003.

3. Mashour NH, Lin GI, Frishman WH. Herbal medicine for the treatment of cardiovascular disease: clinical considerations. Arch Int Med. 1998; 158(20):2225-2234.

4. Monograph. Cratageus oxycantha (Hawthorn) Monograph. Altern Med Rev. 2010;15(2):164-167. 
5. Veveris M, Koch E, Chatterjee SS. Crataegus special extract WS 1442 improves cardiac function and reduces infarct size in a rat model of prolonged coronary ischemia and reperfusion. Life Sci. 2004;74: 1945-1955.

6. Miller AL. Botanical influences on cardiovascular disease. Altern Med Rev. 1998;3(6):422-431.

7. Huang KC. The Pharmacology of Chinese Herbs. 2nd ed. Boca Raton, FL: CRC Press; 1999.

8. Pittler MH, Schmidt K, Ernst E. Hawthorn extract for treating chronic heart failure: meta-analysis of randomized trials. Am J Med. 2003; 114(8):665-674.

9. Shanthi S, Parasakthy K, Deepalakshmi PD, Devaraj SN. Hypolipidemic activity of tincture of Crataegus in rats. Indian J Biochem Biophys. 1994;31:143-146.

10. Kurcok A. Ischaemia and reperfusion-induced cardiac injury: effect of two flavonoid containing plant extracts possessing radical scavenging properties. Naunyn Schmiebergis Arch Pharmacol. 1992;345(Suppl):R81.

11. Zapatero JM. Selections from current literature: effects of Hawthorn on the cardiovascular system. Family Pract. 1999;16(5):534-538.

12. Von Eiff M, Brunner H, Haegeli A, et al. Hawthorn/Passionflower extract and improvement in physical capacity of patients with dyspnoea Class II of the NYHM functional classification. Acta Therapeutica. 1994;20:47-66.

13. Degenring FH, Suter A, Weber M, Saller R. A randomised double blind placebo controlled clinical trial of a standardised extract of fresh Crataegus berries (Crataegisan) in the treatment of patients with congestive heart failure NYHA II. Phytomedicine. 2003;10 363-369.

14. Rietbrock N, Hamel M, Hempel B, Mitrovic V, Schmidt T, Wolf GK. Wirksamkeit eines standardisierten Extraktes aus frischen CrataegusBeern auf Belastungstoleranz und Lebernsqualitat bei Patienten mit Herzinsuffizienz (NYHA II) [A randomised double blind placebo controlled clinical trial of a standardised extract of fresh Crataegus berries (Crataegisan) in the treatment of patients with congestive heart failure NYHA II]. Arzneim-Forsch/Drug Res. 2001;51(II):793-798. German.

15. Tauchert M, Gildor A, Lipinski J. High-dose Crataegus extract in the treatment of NYHA stage II heart failure. Herz. 1999;24(6) 465-474.

16. Tauchert M. Efficacy and safety of crataegus extract WS 1442 in comparison with placebo in patients with chronic stable New York Heart Association class-III heart failure. Am Heart J. 2002;143(5):910-915.

17. Weikl VA, Assmus KD, Neukum-Schmidt A, et al. Crataegus special extract WS 1442, assessment of objective effectiveness in patients with heart failure. Fortschr Med. 1996;114:291-296. German [abstract in English].

18. Valli G, Giardina EG. Benefits, adverse effects and drug interactions of herbal therapies with cardiovascular effects. J Am Coll Cardiol. 2002; 39(7): 1083-1095.

19. Pittler MH, Guo R, Ernst E. Hawthorn extract for treating chronic heart failure. Cochrane Database Syst Rev. 2008;23(1):CD005312.

20. Tyler VE. Herbs of Choice: The Therapeutic Use of Phytomedicinals. New York, NY: Pharmaceutical Product Press; 1994.

21. Schroder D, Weiser M, Klein P. Efficacy of a homeopathic Crataegus preparation compared with usual therapy for mild (NYHAII) cardiac insufficiency: results of an observational cohort study. Eur J Heart Failure. 2003;5:319-326.

22. Asher GN, Viera AJ, Weaver MA, Domink R, Caughey M, Hinderliter AL Effect of hawthorn standardized extract on flow mediated dilation in prehypertensive and mildly hypertensive adults: a randomized, controlled cross-over trial. BMC Complement Altern Med. 2012;12:26.

23. Belz GG, Butzer R, Gaus W, Loew D. Camphor-Crataegus berry extract combination dose-dependently reduces tilt induced fall in blood pressure in orthostatic hypotension. Phytomedicine. 2002;9(7):581-588.

24. Narkiewicz K, Cooley RL, Somers VK. Alcohol potentiates orthostatis hypotension: implications for alcohol related syncope. Circulation. 2000;101(4):398-402.
25. Siegel G, Casper U, Schnalke F, Hetzer R. Molecular physiological effector mechanisms of hawthorn extract in cardiac papillary muscle and coronary vascular smooth muscle. Phytotherapy Res. 1996;(Suppl 10):S195-S198.

26. Bahorun T, Trotin F, Pomery J. Antioxidant activities of Crataegus monogyna extracts. Planta Med. 1994;60:323-328.

27. Bahorun T, Gressier B, Trotin F, et al. Oxygen species scavenging activity of phenolic extracts from hawthorn fresh plant organs and pharmaceutical preparations. Arzneimittelforschung. 1996; 46(II).

28. Vibes J, Lasserre B, Gleye J, Declume C. Inhibition of thromboxane A2 biosynthesis in vitro by the main components of Crataegus oxyacantha (Hawthorn) flower heads. Prostglandins Leukot Essent Fatty Acids. 1994;50:173-175.

29. Zeng T, Guo FF, Zhang CL, et al. A meta-analysis of randomized, double-blind, placebo-controlled trials for the effects of garlic on serum lipid profiles. J Sci Food Agric. 2012;92(9):1892-1902.

30. Reinhart KM, Coleman CI, Teevan C, Vachhani P, White CM. Effects of garlic on blood pressure in patients with and without systolic hypertension: a meta-analysis. Ann Pharmacother. 2008;42(12):1766-1771.

31. Ried K, Frank OR, Stocks NP, Fakler P, Sullivan T. Effect of garlic on blood pressure: a systematic review and meta-analysis. BMC Cardiovasc Disord. 2008;8:13.

32. Rahman K, Lowe GM. Garlic and cardiovascular disease: a critical review. J Nutr. 2006;136(Suppl 3):S736-S740.

33. Li G, Shi Z, Jia H. [Effect of garlicin on unstable angina pectoris and its relationship with blood lipid and GMP-140]. Zhongguo Zhong Yi Jie He Za Zhi. 1998;18(4):208-211. Chinese.

34. Li H, Shi Z, Yusanyin M. [Effects of garlicin tablet on plasma endothelin and nitric oxide in patients with coronary heart disease and angina pectoris]. Zhongguo Zhong Xi Yi Jie He Za Zhi. 1999;19(11):670-671. Chinese.

35. Li G, Shi Z, Jia H, et al. [A clinical investigation on garlicin injection for treatment of unstable angina pectoris and its actions on plasma endothelin and blood sugar levels]. J Trad Chin Med. 2000;20(4): 243-246. Chinese.

36. McKenna DJ, Jones K, Hughes K. Efficacy, safety, and use of gingko biloba in clinical and preclinical applications. Altern Ther Health Med. 2001;7(5):70-86.

37. Mahadevan S, Park Y. Multifaceted therapeutic benefits of Ginkgo biloba L: chemistry, efficacy, safety, and uses. J Food Sci. 2008;73(1): R14-R19.

38. Schultz V, Hansel R, Typer VE, editors. Rational Phytotherapy: A Physician's Guide to Herbal Medicine. 4th ed. Berlin, Germany: Springer; 2001:171-175.

39. Kuller LH, Ives DG, Fitzpatrick AL, et al. Does Ginkgo biloba reduce the risk of cardiovascular events? Circ Cardiovasc Qual Outcomes. 2010;3(1):41-47.

40. Srivastava RD, Dwivedi S, Sreenivasan KK. Chandrashekhar CN. Cardiovascular effects of Terminalia species of plants. Indian Drugs. 1992;29:144-149.

41. Maulik SK, Katiyar CK. Terminalia arjuna in cardiovascular diseases: making the transition from traditional to modern medicine in India. Curr Pharm Biotechnol. 2010;11(8):855-860.

42. Artham SM, Lavie CJ, Milani RV, Patel DA, Verma A, Ventura HO. Clinical impact of left ventricular hypertrophy and implications for regression. Prog Cardiovasc Dis. 2009;52(2):153-167.

43. Dwivedi S, Agarwal MP. Antianginal and cardioprotective effects of Terminalia arjuna, an indigenous drug, in coronary artery disease. J Assoc Physicians India. 1994;42(4):287-289.

44. Kumar PU, Adhikari P, Pereira P, Bhat P. Safety and efficacy of Hartone in stable angina pectoris - an open comparative trial. JAssoc Physicians India. 1999;47(7):685-689.

45. Bharani A, Ganguli A, Mathur LK, Jamra Y, Raman PG. Efficacy of Terminalia arjuna in chronic stable angina: a double-blind, placebocontrolled, crossover study comparing Terminalia arjuna with isosorbide mononitrate. Indian Heart J. 2002;54(2):170-175. 
46. Lockhande PD, Dhaware BS, Jagdale SC, Chabukswar AR, Mulkalwar SA. Cardiac activity of isolated constituents of Inula racemosa. $J$ Herbal Pharmocotherapy. 2006;6(3-4):81-88.

47. Ojha S, Nandave M, Kumari S, Arya DS. Cardioprotection by Inula racemosa Hook in experimental model of myocardial ischemic reperfusion injury. Indian J Experimental Biology. 2010;48(9):918-924.

48. Tripathi SN, Upadhyaya BN, Gupta VK. Beneficial effect of Inula racemosa (pushkarmoola) in angina pectoris: a preliminary report. Indian J Physiol Pharmacol. 1984;28(1):73-75.

49. Dwivedi S, Somani PN, Udupa KN. Role of Inula racemosa and Saussurea lappa in management of angina pectoris. Pharm Bio. 1989; 27(4):217-222.

50. Bensky D, Clavey S, Stoger E. Chinese Herbal Medicine Materia Medica. 3rd ed. Seattle, WA: Eastland Press; 2004.

51. Wongcharoen $\mathrm{W}$, Phrommintikul A. The protective role of curcumin in cardiovascular diseases. Int J Cardiol. 2009;133(2):145-151.

52. Alwi I, Santoso T, Suyono S, et al. The effect of curcumin on lipid level in patients with acute coronary syndrome. Acta Med Indones. 2008; 40(4):201-210.

53. Adams JD, Wang R, Yang J, Lien EJ. Preclinical and clinical examinations of Salvia miltiorrhiza and its tanshinones in ischemic conditions. Chin Med. 2006;1:3. Available from: http://www.cmjournal. org/content $/ 1 / 1 / 3$. Accessed June 12, 2012.

54. Bensky D, Gamble A. Chinese Herbal Materia Medica. Washington: Eastland Press; 1993.

55. Pharmacopoeia Commission of PR China. Pharmacopoeia of the People's Republic of China (English edition). Guangzhou, China: Guangdong Science and Technology Press; 1992.

56. Ji XY, Tan BKH, Zhu YZ. Salvia miltiorrhiza and ischemic diseases. Acta Pharmacol Sin. 2000;21:1089-1094.

57. Frishman WH, Sinatra ST, Moizuddin M. The use of herbs for treating cardiovascular disease. Sem Integrat Med. 2004;2(1):23-35.

58. Xie M, Gu Z, Chen K. Progress in experimental research of Radix Salviae miltiorrhizae, Fructus Crataegi and their components on atherosclerosis. Chinese J Integr Med. 1998;4(4):311-314.

59. Tao Y-Y, Guo S-D, Chen H-Z, et al. Effects of compound salviae miltiorrhizae injection on metabolism of phosphoinosititides in myocardial ischemia. Chinese J Integr Med. 1995;1(4):288-290.

60. Jiang RW, Lau KM, Hon PM, Mak TCW, Woo KS, Fung KP. Chemistry and biological activities of caffeic acid derivatives from Salvia miltiorrhiza. Curr Med Chem. 2005;12:237-246.

61. Zhou W, Ruigrok TJ. Protective effect of danshen during myocardial ischemia and reperfusion, an isolated rat heart study. Am J Chin Med. 1990;18(1-2):19-24.

62. Fung KP, Zeng LH, Wu J, et al. Demonstration of the myocardial salvage effect of lithospermic acid B isolated from the aqueous extract of Salvia miltiorrhiza. Life Sci. 1993;52(22):L239-L244.

63. Wu YJ, Hong CY, Lin SJ, Wu P, Shiao MS. Increase of vitamin $\mathrm{E}$ content in LDL and reduction of atherosclerosis in cholesterol-fed rabbits by a water soluble antioxidant-rich fraction of Salvia miltiorrhiza. Arterioscler Throm Vasc Biol. 1998;18: $481-486$.

64. Huang JL, Chen Y, Huang YQ. Induction of vascular endothelial growth factor genes expression by Dan Shen (Radix Salviae miltiorrhizes) in human fibroblasts. Available from: http://www.ustcma.org/AJTCM/ vol1-2-2000/JanLi-HUANG.htm. In: Sun J, Tan BKH, Huang S-H, Whiteman M, Zhu Y-Z. Effects of natural products on ischemic heart diseases and cardiovascular system. Acta Pharmacol. 2002;23(12): $1142-1151$.

65. Sun X, Chan LN, Sucher NJ. Magnesium as NMDA receptor blocker in the traditional Chinese medicine Danshen. Phytomedicine. 2004;12(3):173-177.

66. Lei X-L, Chiou GCY. Cardiovascular pharmacology of Panax notoginseng (Burk) FH Chen and Salvia miltiorrhiza. Am J Chin Med. 1986;14(3-4):145-152.

67. Lei X-L, Chiou GCY. Studies on cardiovascular actions of Salvia miltiorrhiza. Am J Chin Med. 1986;14(1-2):26-32.
68. Kamata K, Noguchi M, Nagai M. Hypotensive effects of Lithospermic Acid B isolated from the extract of Salviae Miltiorrhizae Radix in the rat. Gen Pharmac. 1994;25(1):69-73.

69. Kang DG, Oh H, Chung HT, Lee HS. Inhibition of angiotensin converting enzyme by lithospermic acid B isolated from radix Salviae miltiorrhiza Bunge. Phytother Res. 2003;17:917-920.

70. Kamata K, Lizuka T, Nagai M, Kasuya Y. Endothelium-dependent vasodilator effects of the extract from Salviae miltiorrhizae Radix. A study on the identification of Lithospermic Acid B in the extracts. Gen Pharmac. 1993;24(4):977-981.

71. Tang M-K, Ren D-C, Zhang J-T, Du G-H. Effects of salvianolic acids from Radix Salviae miltiorrhizae on regional cerebral blood flow and platelet aggregation in rats. Phytomedicine. 2002;9: 405-409.

72. Li M, Zhao C, Wong RNS, Goto S, Wang Z, Liao F. Inhibition of shear-induced platelet aggregation in rat by tetramethylpyrazine and salvianolic acid B. Clin Hemorheol Microcirc. 2004;31(2): 97-103.

73. Ni J, Liu G, Wei J, Qiao J, et al. Chinese medicinal herb "Dan Shen" compound for acute myocardial infarction [Protocol for a Cochrane Review]. Cochrane Library Issue 1. Chichester: John Wiley \& Sons; 2004

74. Chen Y, Lin S, Ku H, Shiao M, Chen J, Chen Y. Salvianolic acid B attenuates VCAM-1 and I-CAM 1 expression in TNF-alpha-treated human aortic endothelial cells. J Cell Biology. 2001;82:512-521.

75. Liu JR, Chen GF, Shih HN, Kuo PC. Enhanced antioxidant bioactivity of Salvia miltiorrhiza (Danshen) products prepared using nanotechnology. Phytomedicine. 2008;15(1-2):23-30.

76. Zhang K, Bao Y, Wu P, Rosen R, Ho C. Antioxidative components of Tanshen (Salviae miltirrhiza Bunge). J Agric Food Chem. 1990;38: 1194-1197.

77. Ho JH-C, Hung C-Y. Salvianolic acids: small compounds with multiple mechanisms for cardiovascular protections. J Biomed Sci. 2011; 18:30.

78. Li Z, Zhu L, Huang B. Effects of purified herbal extract of Salvia miltiorrhiza on lipid profile in hyperlipidemic patients. $J$ Geriatr Cardiol. 2009;6(2):99-101.

79. Shanghai Cooperative Group for the Study of Tanshinone IIA. Therapeutic effect of sodium tanshinone IIA sulfonate in patients with coronary heart disease. J Trad Chin Med. 1984;4:20-24.

80. Shanghai Clinical Cooperation Group of Danshen 201. [The effects of tanshinone IIA (sodium sulfonate) injection of the treatment of 108 patients with angina pectoris]. Commun J Chin Herbal Med. 1979;1:37-39. Chinese.

81. Ou M. Chinese-English Manual of Common-Used in Traditional Chinese Medicine. Guangzhou, China: Guangong Science and Technology Press; 1992.

82. Gao H, Wang F, Lien EJ, Trousdale MD. Immunostimulating polysaccharides from Panax notoginseng. Pharm J. 1996;13(8): $1196-1200$

83. Lin SG, Zheng XL, Chen QY, Sun JJ. Effect of Panax notoginseng saponins on increased proliferation of cultured aortic smooth muscle cells stimulated by hypercholesterolemic serum. Zhongguo Yao Li Xue Bao. 1993;14:314.

84. Li SH, Chu Y. [Anti-inflammatory effects of total saponins of Panax notoginseng]. Zhongguo Yao Li Xиe Bao. 1999;20(6):551-554. Chinese.

85. Chan P, Thomas GN, Tomlinson B. Protective effects of Trilinolein extracted from Panax notoginseng against cardiovascular disease. Acta Pharmacol Sin. 2002;23(12):1157-1162.

86. Lang J, Cao H, Wei A. [Comparative study on the effect of Panax notoginseng and ticlid in treating early diabetic nephropathy]. Zhongguo Zhong Xi Yi Jie He Za Zhi Zhongguo Zhongxiyi Jiehe Zazhi. 1998;18(12):727-729. Chinese.

87. Chan SS-K, Cheng T-Y, Lin G. Relaxation effects of ligustilide and senkyunolide A, two main constituents of Ligusticum chuanxiong, in rat isolated aorta. J Ethnopharmacol. 2006;111(3):677-680. 
88. Hou J, He X. Research development on volatile oil from chuanxiong rhizome. J Med Plant Res. 2012;6(12):2240-2248.

89. Xiao P-G, Liu C-X. Pharmacology, pharmacokinetics and toxicology of Chinese traditional medicine for stroke therapy. Asian Journal of Drug Metabolism and Pharmacokinetics. 2005;5(2):83-124.

90. Teng J, Chen H, Li D, Luo A. Identification and characterization of supercritical fluid extracts of Rhizoma Chuanxiong by high performance liquid chromatography ion trap mass spectrometry. Frontiers of Chemistry in China. 2006;1(4):454-458.

91. Chan SS-K, Jones RL, Lin G. Synergistic interaction between the Ligusticum chuanxiong constituents butylidenephthalide and the nitric oxide donor sodium nitroprusside in relaxing rat isolated aorta. J Ethnopharmacol. 2009;122(2):308-312.

92. Lu C-H. A study of the effect of extract of Ligusticum chuanxiong Hort on isolated rat thoracic aorta and its possible mechanism. Heilongjiang Medical Journal. 2008. Available from: http://www.en.cnki.com.cn/Article_en/ CJFDTOTAL-HLYX200802018.htm. Accessed June 14, 2012.

93. Zhou Y, Hu P-C, Deng P-Y, Deng H-W, Li Y-J. The protective effects of ligustrazine on ischaemia-reperfusion and DPPH free radical-induced myocardial injury in isolated rat hearts. Planta Med. 2004;70(9):818-822.

94. Liu L, Ning Z-Q, Shan S, et al. Phthalide lactones from Lingusticum chuanxiong inhibit lipopolysaccharide-induced TNF-alpha production and TNF-alpha-mediated NF-kappaB activation. Planta Med. 2005; 71(9):808-813.

95. Zhang YH, Rose K. A Brief History of Qi. Brookline: Paradigm Publications; 2001.

96. Cai J, Chao G, Chen D, Chen X, Cheng X, Dong L, editors. Advanced Textbook on Traditional Chinese Medicine and Pharmacology. Beijing, China: New World Press; 1995.

97. Beinfeld H, Korngold E. Between Heaven and Earth. A Guide to Chinese Medicine. New York, NY: Ballantine Books; 1991.

98. O'Brien KA, Xue CCL. The theoretical framework of Chinese medicine. In: Leung P-C, Xue CC, Cheng Y-C, editors. A Comprehensive Guide to Chinese Medicine. Singapore: World Scientific Press; 2003.

99. O’Brien KA, Luo RZ. Chinese medicine perspectives on myocardial ischaemia. In: Vukovic D, Kiyan V, editors. Myocardial Ischemia: Causes, Symptoms and Treatment. New York City, NY: Nova Science Publishers; 2009:149-193.

100. Maciocia G. The Foundation of Chinese Medicine. New York: Churchill Livingstone; 1989.

101. Ni M. The Yellow Emperor's Classic of Medicine. A New Translation of the Neijing Su Wen with Commentary. Boston: Shambala Publications; 1995.

102. Li-Ling J. The Jing-Mai connections of the Heart. Int J Cardiol. 2003; 89:1-11.

103. O'Brien KA, Birch S. A review of the reliability of diagnosis in traditional East Asian medicine. J Altern Comp Med. 2009;15(4):353-366.

104. Jiang W-Y. Therapeutic wisdom in traditional Chinese medicine: a perspective from modern science. Trends Pharmacol Sci. 2005; 26(11):558-563.

105. Wang J, Jing L, Zhong JB, Wang YY, Ma LH, Liu JG. [Clinical study on compatibility and dismantlement of Xuefu Zhuyu decoction]. Zhongguo Zhong Yao Za Zhi (China Journal of Chinese Materia Medica). 2004;29(8):803-807. Chinese.
106. Scheid V. Shaping Chinese medicine: two case studies from contemporary China. In: Hsu E, editor. Innovation in Chinese Medicine. Cambridge, UK: Cambridge University Press; 2001.

107. Clavey S. Fluid Physiology and Pathology in Traditional Chinese Medicine. London: Churchill Livingstone; 2004.

108. Zhuo Q, Yuan Z, Chen H, Wu T. Traditional Chinese herbal products for stable angina [review]. Cochrane Database Syst Rev. 2010; 5:CD004468.

109. Bensky D, Barolet R. Chinese Herbal Medicine Formulas and Strategies. Seattle, WA: Eastland Press; 1990.

110. Yu S, Zhong B, Zheng M, Xiao F, Dong Z, Zhang H. The quality of randomized controlled trials on DanShen in the treatment of ischemic vascular disease. J Altern Complem Med. 2009;15(5): $557-565$.

111. Fang XM, Xiao LH, Yang JS. [Yi xin mai granules for 30 cases of CHD stable angina]. Shanxi Zhong Yi (Shangxi J Trad Chinese Med). 2002;23(12):1092-1093. Chinese.

112. Wang AC, KE YS, Yang SY. Clinical observation of "Bao Xin Bao" patch for 40 cases of CHD stable angina. Modern J Integrated Chinese Traditional and Western Medicine. 1999;8(9):1412-1413.

113. Wang SS, Zhang CL, Han D. Clinical observation of "Li Nao Xin" capsule for 80 cases of CHD angina. Chinese J Gerentology. 2004; 24:1196.

114. Wang G, Wang L, Xiong ZY, Mao B, Li TQ. Compound salvia pellet, a traditional Chinese medicine, for the treatment of chronic stable angina pectoris compared with nitrates: a meta-analysis. Med Sci Monit. 2006;12(Suppl 1):SR1-SR7.

115. Yan SY, Yao C, Ding XR, Jiang M, Lu AP. Serum low-density lipoprotein and heart rate related to the effectiveness in the treatment of angina pectoris with two similar Chinese herbal formulae. Chin J Integr Med. 2011;17(12):903-907.

116. Gao L, Li YJ, Chen KY. [Effects of supplementing qi and activating blood circulation method on platelet aggregation rate, adhesion rate and thromboxane B2 level in patients with stable angina pectoris and intolerable to aspirin]. Zhongguo Zhong Xi Yi Jie He Za Zhi. 2008; 28(4):300-303. Chinese.

117. Wu H-L, Wang X, Li X-M, Luo W-J, Deng T-T. Trial study on Deng Tie-tao's Coronary Heart Disease Capsules in improving patients' quality of life. Chin J Integr Med. 2005;11(3):173-178.

118. Liao X, Luo L. [Clinical observation of yugengongyu decoction in treatment of coronary heart disease with angina pectoris]. Zhongguo Zhong Xi Yi Jie He Za Zhi. 1998;18(10):594-597. Chinese.

119. Chen K. [The therapeutic effect of purified coronary heart II tablets on 112 cases of angina pectoris by double blind methods]. Zhonghua Xinxueguanbing Zazhi. 1982;10:81-89. Chinese.

120. Shanghai University Physiology Department. [Therapeutic effects of danshen injection on coronary heart disease]. Chin J Intern Med. 1997;4:203-206. Chinese.

121. Zhang AL, Story DF, Lin V, et al. A population survey on the use of 24 common medicinal herbs in Australia. Pharmacoepidemiol Drug Saf. 2008;17(10):1006-1013.

Botanics: Targets and Therapy

\section{Publish your work in this journal}

Botanics: Targets and Therapy is an international, peer-reviewed, open access journal focusing on the discovery and development of active compounds based upon or found naturally occurring in the plant kingdom that may have therapeutic potential in any disease state. The manuscript management system is completely online and includes a very

\section{Dovepress}

quick and fair peer-review system. Visit http://www.dovepress.com/ testimonials.php to read real quotes from published authors. 